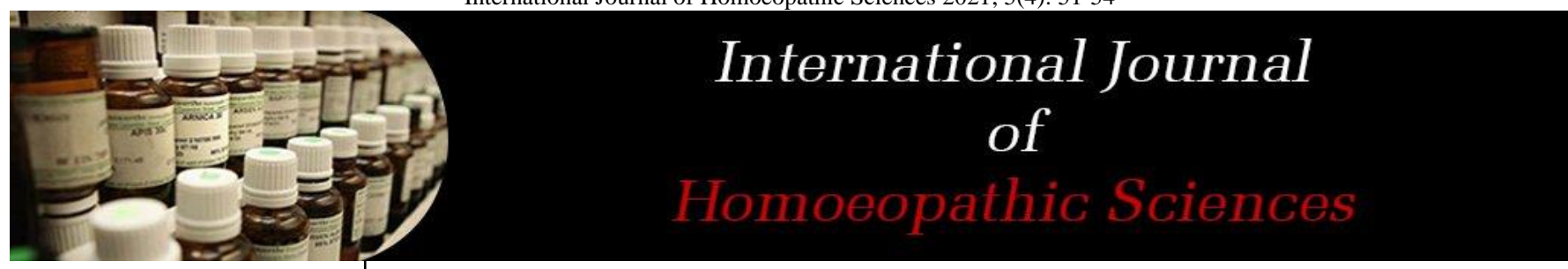

E-ISSN: 2616-4493 P-ISSN: 2616-4485 www.homoeopathicjournal.com IJHS 2021; 5(4): 51-54

Received: 25-08-2021

Accepted: 27-09-2021

Dr. Yamuna Tenka Department of Pharmacy, Guru Mishri Homoeopathic, Medical College, University of Maharashtra Health Science, Maharashtra, India
Corresponding Author: Dr. Yamuna Tenka Department of Pharmacy, Guru Mishri Homoeopathic, Medical College, University of Maharashtra Health Science, Maharashtra, India

\section{Sarcodes and its homoeopathic application}

\section{Dr. Yamuna Tenka}

DOI: $\underline{\text { https://doi.org/10.33545/26164485.2021.v5.i4a.453 }}$

\section{Abstract}

The healing art process comes from within. It is the physician's responsibility to start from the source and not from the patient. This review briefly describes the various properties of sarcodes and their effects on humans homeopathically.

Keywords: sarcodes, organotherapy, homeopathic remedy, organ extract

\section{Introduction}

Sarcodes are homeopathic drugs that are prepared from the secretions and tissues of healthy animals. They contain biological molecules that have physiological functions in humans.

The codes contain information about an organ's biological structure and its energy-producing substance. They are used to remove toxins and improve organ function.

Sarcodes are extracted from animal or human tissues and used to treat various conditions. They are also known as cellular homoeopathy.

The use of sarcodes helps transfer genetic information from the nucleus to the cytoplasm of cells. It serves as a template for the synthesis of protein.

\section{History}

Various organs of animals have been used for medical purposes. Although it is not yet widely known that they were used during ancient times, the use of orchitic substances for the treatment of obesity was noted down by Sushruta, who lived sometime between 400 and 500 B. C. This is probably the first mention of Organotherapy and from that time, frequent references have been made in literature.

Sarcodes and nosodes are groups of drugs that were added to the homeopathic medicine in the 1830s. Most of the substances used in these regimens have a long medical tradition.

This type of homeopathy was introduced in the 1930s by the English Physician, Dr. Conan, for the treatment of acute or chronic diseases of the digestive system. It was later extended to other pathologies. Next, a French physician Dr. Nobel used secretions of insulin hormone produced by the pancreas for the treatment of diabetes. He later used other substances for the treatment of a variety of conditions with great success. Immediately after this introduction, Organ homeopathy became widely used due to its simplistic approach and phenomenal results

\section{Definition and its function in the body}

The treatment of diseases with the use of sarcodes derived from animal organs is known as organotherapy. This procedure is usually carried out by using a combination of therapeutic agents and procedures.

Animal protoplasm is the term used for the secretion of protoplasm from glands or organs of animals. Few of the functions are mentioned below:

- Balances organs, glands or tissue activity

- Slows down accelerated organs, glands or tissue activity

- Stimulates sluggish organs, glands or tissue activity

- Slows down accelerated organs, glands or tissue activity

- Will up regulate and restore hypothyroid balance OR can down regulate the overall activity of the hyperthyroid gland (using thyroid hormone extract or Thyroidinum)

- In cases where organs are damaged due to autoimmune issues, organotherapy substitutes for the organ and accepts the autoimmune antibodies, leaving the organ to stimulate its own restoration. 


\section{Fundamental principle}

Organotherapy is an up and emerging branch of Homeopathy that utilizes the utilization of organ remedies, made up of the health tissues of animals, to treat the patient, performing on the basic principles of Homeopathy, i.e. like cures like. It is supported the thought and understanding that organs answer those tissues that have an affinity for an equivalent tissues within the physical body, regardless of what species their origin. Organotherapy is based on two fundamental laws.

(1) Identical organs.

(2) Activity by potency.

1. Identical organs: When a diseased organ is present, an identical healthy organ extract with corrected information in the form of mRNA is administered to rectify the organ.

2. Activity by potency: Organ medicine is used to strengthen organs, glands, and tissues weakened by toxins. A low potency is used to stimulate an underactive organ, gland, or tissue.

\section{How it works}

Sarcodes have a very notable peculiarity. They always exist in molecular form within the organism, and participate in various molecular interactions being a part of different biochemical pathways. They become homeopathic drugs only when they are not administered in ,molecular forms ${ }^{\text {ee }}$, but as potentized forms above 12c. In molecular forms below Avogadro limit, they will be considered only as physiological products, not as homeopathic drugs.

\section{General Mechanism}

It stimulates organ functions and should help to stop antibodies from being formed against those particular organs. The organs can recognize the proper cells and can start reproducing them from the Organotherapy stimulation. The response of a malfunctioning or diseased organ to the Organotherapy (sarcode) remedy is that the function of the organ or tissue is then supported in its healing, regulation and balance. Organotherapy sarcodes also can act as if to exchange an organ which was removed partially or totally with surgery like women with a hysterectomy surgery, or removal of their ovaries.

It is also wont to help with organ drainage so as to revive function to organs that are suffering from progressing disease. This may be especially important for the kidneys and liver.

\section{Hormonal Mechanism}

Sarcodes having hormonal origin undergo two sorts of chemical interactions: 1. "On-target interactions" and a couple of "Off-target interactions" "On target interactions" are essential parts of important processes occurring between natural ligands and their genuine targets. They're of two types, 1. Molecular identification and binding, 2. Actual chemical interactions. "Off target interactions" are people who occur between ligands and wrong targets i.e. inhibitory pathways. As sarcodes have molecular imprints they act as interfering agents in off targeting interactions. E.g. Thyroidinum 30 .

\section{Characteristics}

1. Sarcodes belong to the less proved group of remedies.

2. Sarcodes are mainly stated to be the organopathic drugs.

3. All sarcodes are deep acting remedies.

4. Deficient functioning or hyper functioning of organs.

5. Symptoms are related to sources of preparation.

6. Headache worse on waking up in the morning, throbbing with nausea and vomiting.

7. Obstinate constipation. 8. Complaints are aggravation due to intake of milk lac can, lac dul,

8. Nervousness

9. Profuse urination with increased thirst. So they can be used in diabetes

10. Obesity,

11. Malignancy, Tendency to over growth

\section{Preparation}

The steps have been described here with for sarcode preparation method in Table 1 .

Table 1: Steps of sarcode preparation

\begin{tabular}{|c|c|}
\hline Step 1: nature of material & $\begin{array}{l}\text { Sources: } \\
\text { 1. from whole endocrine glands } \\
\text { 2. from healthy secretions } \\
\text { 3. from extract } \\
\text { 4. other sarcodes } \\
\end{array}$ \\
\hline Step 2: safety & $\begin{array}{l}\text { Operation is carried out in stringent biosafety compliant environment with minimum handling using sealed } \\
\text { containers and disposable auto-tip pipettes }\end{array}$ \\
\hline $\begin{array}{l}\text { Step 3: Removal and } \\
\text { separation of other } \\
\text { components }\end{array}$ & $\begin{array}{l}\text { - Filtration (Seitz filter) is done to get rid of cell debris, unidentified bacteria and large protein particles. } \\
\text { - If the source material is obtained as scraping of animal or human tissues, the keratin component of skin is } \\
\text { removed by boiling the scrapings with potassium hydroxide }(\mathrm{KOH}) \text { in water medium. }\end{array}$ \\
\hline $\begin{array}{l}\text { Step 4: preparation of } \\
\text { mother tincture }\end{array}$ & $\begin{array}{l}\text { - Soluble substances are infused directly in alcohol or in an alcohol/water mixture. This process is called } \\
\text {,trituration"e } \\
\text { - Trituration proceeds until the ingredient has become fine enough to be soluble in alcohol or in both cases is } \\
\text { known as the mother tincture. }\end{array}$ \\
\hline $\begin{array}{l}\text { Step 5: Dynamizaton of } \\
\text { potencies }\end{array}$ & $\begin{array}{l}\text { - } 1 \text { part of the mother tincture is diluted in } 99 \text { parts of alcohol or in a mixture of water and alcohol. } \\
\text { - This liquid is then succussed } 10 \text { times in its bottle by firmly hitting the bottlees base against a firm but } \\
\text { resistive surface such as the palm of a hand or leather covered over book. The resulting liquid is called 1C } \\
\text { potency ( } 1 \text { refers to } 1 \text { st stage of dilution and C refers to its } 1: 1000 \text { dilution ratio). } \\
\text { - 1part of this } 1 \mathrm{C} \text { potency is again diluted and succussed in } 99 \text { parts of alcohol or water and alcohol mixture to } \\
\text { produce a } 2 \mathrm{C} \text { remedy. } \\
\text { - This serial of process of dilution and succession, called potentisation, is further repeated to produce } \\
\text { increasingly higher potencies of the remedy. }\end{array}$ \\
\hline Step 6: Safety check for & Test for sterility for aerobic and anaerobic organism should be done before issue of any sarcode, for \\
\hline
\end{tabular}




\begin{tabular}{|c|c|}
\hline human use & therapeutic use or for manufacturing of higher attenuation \\
\hline Step 7: Lyophilization & $\begin{array}{c}\text { Lyophilization of the original stock is done so that sarcodes can be prepared in future, without any need to } \\
\text { repeat initial steps. A centralized depository system preserves standardized raw material for future use }\end{array}$ \\
\hline
\end{tabular}

\section{Indications}

- As a complementary to the constitutional remedy - both as an acute or chronic e.g. Thyroidinum as an acute of Natrum mur in an acute case of urticaria or Thyroidinum as a deeper acting constitutional remedy in an acute case of angina which was treated with Adrenalinum.

- As an organ stimulator when the affected organ is functionally or structurally affected e.g. Pituitarium glandula as an intercurrent organ remedy during a case of pituitary microadenoma

- Clinical conditions, where the organ loses its very function or structure or conditions characterized by atrophy e. g. cirrhosis of liver, medulla spinalis neoplasm, cerebellar degeneration etc. and one can prescribe Hepar suis, spinal cord or Cerebellum respectively in such cases.

- Clinical conditions characterized by hyper functioning of organs e.g. adrenal hyper function (like Cushing syndrome, cortex adenoma or cortex carcinoma etc.) where one can use a sarcode like Adren. it's going to be overgrowth, acromegaly or obesity. On an equivalent analogical thinking, malignant pathologies also come under pathogenesis of sarcodes.

- When one organ is that the basis of the overall condition of great order, a prescription of a sarcode are often thought of e.g. if a tolerant myocardial ischemia has now CCF and constitutional and organ remedies supported indications aren't helping the case, a sarcode like Myocardium are often thought of or if one diseased organ of kidney has caused systemic effects on the system, one use a sarcode like Ren or an inveterate case that has suffered from multiple skin conditions (from simple dermatitis to psoriasis for example) throughout the lifetime of a patient and which has baffled many good prescriptions, a sarcode like Derma are often prescribed.

- Paucity of symptoms during a case of either functional or structural type where it's difficult to seek out a constitutional remedy.

- A case of pathological museum where multiple vital organs are affected within the system. One can use many sarcodes during the treatment span, not together but singly during a sequential way.

- The disease shows its ,play ${ }^{\text {ee }}$ in some organs that are bound together by a system e.g. a mixture of DM, hypothyroidism and PCOD during a case where endocrinological system is affected.

- Exorbitant investigatory reports during a functional or structural case e.g. high cholesterol, high bilirubin, high creatinine or blood urea etc.

- established order condition during a pathological case when nothing, „movese towards recovery after the administration of several remedies.

- On the past and case history of certain clinical conditions which may have a dynamic effect over the constitution e.g. history of hypo/hyper-thyroidism in case history Preponderance of structural zone of illness with low susceptibility. $\neg$ or during pregnancy where one can prescribe Thyroidinum.

- A case of advanced pathological case where the vitality is at low ebb and it's inappropriate to prescribe a constitutional remedy because it may end in a killer aggravation. Preference should tend to a sarcode than a nosode in such cases because the fuse are often burnt with a nosode.

- Clinical confirmation are often a source of use of a sarcode e.g. Pituitrin in conditions of delayed puberty and undeveloped breasts.

- For the sake of detoxification

\section{Classification}

1. Based on source of sarcodes

Sarcodes are prepared from.

A. Healthy endocrine glands as a whole.

B. Normal secretion from endocrine glands.

C. Normal secretions from endocrine glands.

D. Product (or extract) of animal glands and tissues.

E. Healthy organs of animals.

F. Others

\section{Based on use of frequency}

A. Those which are of well-known and practically unquestioned therapeutic efficacy.

B. Those which aren't documented but which, nevertheless, offer undoubted possibilities in medicine.

C. Those which are used slightly, which are occasionally mentioned in medical literature, but so far have a relatively limited usage.

\section{Sarcodes and human symbolic language}

In universe, everything which exists outside of man has its analogue within man. a person beholds partially that celestial effulgence (shining forth brilliantly) I which all creation is bathed. Each emotion features a related organ or organs. Emotions are capable of causing physiological and anatomical alterations in organs. So, looking backwards, every condition are often traced back to its emotional origin. Every organ has its secret meaning. Each sarcode remedy carries within it the symbolic language of the human system and of the organs and this might be utilized for developing the theme and for selection of the acceptable sarcode remedy.

Examples: Breast, Colon, pituitary.

- Breast(s): The human symbolic language of breasts represents mothering, nurturing and nourishment. Illnesses (like cysts, lumps, soreness, mastitis etc.) represent refusal to nourish the self and putting everyone else first. Indications of Lac group of remedies (prepared from milk of the many animals) are associated with deprivation of affection, faulty nurturing and forsaken and inferiority feeling.

\section{Conclusion}

The final aim of any physician or healer is to supply or help their patients to regain the homeostatic balance for leading a healthy life. Sarcodes are the quintessential preparations charged with the sar of physical body this provides the 
essential advantage over modern medicine is that these preparations are one among body itself and hence promote the principle of self healing. they're easily accepted by the body system and need no special process as needed for any drug of herbal or allopathic origin. These remedies are often administered in individualistically and in sort of combinations over a broad demographic. These remedies provide innumerable indications to be used along side modern medicines.

\section{References}

1. Chandran KC. Nosodes, Sarcodes, Vaccines- A Comparative Study from MIT Perspective 2015, 11. Redefining Homeopathy. https://dialecticalhomeopathy.wordpress.com/2015/12/1 1/nosodes-sarcodes-vaccines-acomparative-study-frommit-perspective/

2. Dr. Ajit Kulkarni. Sarcodes: Resonating Organ Arsenal. http://homeoint.ru/pdfs/Sarcodes.pdf

3. Homeopathy for women, Organotherapy-Use of sarcodes. https://www.homeopathyforwomen.org/organotherapy. htm

4. My A. International Laboratories, Inc.: Herbal and Homeopathic Remedies and Nutritional Supplements, History and development of Homeopathy; http://herballabs.net/us/homeopathy/history-ofhomeopathy

5. Innovative Medicine, Organotherapy; https://innovativemedicine.com/solutions/organotherap $\mathrm{y} / \mathrm{tab}=3 /$

6. Robert Medhurst. Isopathy, Homeopathic Papers 2010. http://hpathy.com/homeopathy-papers/isopathy-2/

7. Jarvis HG. Organotherapy. The Boston Medical and Surgical Journal 1914;171(4):158-61.

8. Vieracker V. Nosode and sarcode therapies and their history--a controversial inheritance. Medizin, Gesellschaft, und Geschichte: Jahrbuch des Instituts fur Geschichte der Medizin der Robert Bosch Stiftung, 2015;33:155-77.

9. English Oxford Living Dictionary. https://en.oxforddictionaries.com/definition/organothera py 10 . Homeopathic solutions: A scientific and holistic approach of treatment, Knowledge Academy, Organotherapy: https://www.dratiq.com/academy/organo.html.

10. Dr. Merra Narendran. The sarcodes in Homeopathy, March 11, 2012. Homeo Book: everything on Homeopathy.

http://www.homeobook.com/the-sarcodes-inhomeopathy/

11. Dr. Sudheera AP. The repertory of Homoeopathic Nosodes and Sarcodes, April 18, 2013. Similima.com: Everything on Homeopathy. http://www.similima.com/therepertory-ofhomoeopathic-nosodes-and-sarcodes/

12. Homeopathic Remedies \& Treatment; Organotherapy: What is Organotherapy. Adi BS, Adi GB, Jamadade AK. A Comparison of the Efficacy of Gymnema Sylvestre $6 \mathrm{Ch}$ and Gymnema Sylvestre Mother Tincture in Cases of Type 2 Diabetes Mellitus. World Journal of Current Medical and Pharmaceutical Research 2020;1:133-8.

http://homeopathicremediesandtreatment.com/Organoth
erapy-What-isOrganotherapy.php

13. The list of sarcode and nosode remedies in Homeopathy, December 3, Homeo Book: Everything on Homeopathy 2016. http://www.homeobook.com/listof-sarcode-andnosode-remedies-in-homeopathy/

14. Dr. Devendra Kumar. Nosodes and Sarcodes Origin and Source of Homeopathic Medicines; HomeoResearch.com: Searching Homeopathy. http://homeoresearch.blogspot.com/2010/09/nosodesand-sarcodes-origin-andsources.html

15. Utility of Sarcodes in Homeopathy, December 18, Homeo book: Everything on Homeopathy 2012. http://www.homeobook.com/utility-of-sarcodes-inhomoeopathy/

16. Sankar K, Jadhav AP. Nosodes and Sarcodes. Indian journal of traditional knowledge 2017;16(1):158-163.

17. Partha MP, Mandal B. A Text Book of Homoeopathic Pharmacy: B. Jain Publishers, 2001;27-28.

18. Dr. Mansoor Ali. A Study of Nosodes \& Sarcodes in Homoeopathy http://www.homeobook.com/pdf/nosodes-sarcodeshomeopathy.pdf

19. Handbook on healing, Book II; by Swami Narayani and Swami Ananda, Chapter - Gem Colour Healing 137145.

20. Richard Pitt. Sarcodes; Comparative Materia Medica: Integrating New \& Old Remedies. http://www.comparativemateriamedica.com/sarcodes/

21. Dr. Robert Seror, H.M.M. Reconstituted about Nosodes and Sarcodes according to Dr. John Henry Clarke. http://www.homeoint.org/seror/nosodes/sarcodes.htm

22. Homeopathy for women, Matridonal Sarcodes. https://www.homeopathyforwomen.org/matridonal_sar codes.htm

23. Harrower HR. Practical Organotherapy: The Internal Secretions in General Practice: Harrower laboratory 1919, 31-33.

24. Sarcode therapy as a restorative of health, world journal of Pharmacy And Pharmaceutical Sciences, Faculty of Pharmacy, G.H. Patel Building, Donor's Plaza, The M.S. University of Baroda, Vadodara-390002, India. 